\title{
Surface runoff estimation and prediction regarding LULC and climate dynamics using coupled LTM, optimized ARIMA and distributed-GIS-based SCS-CN models at tropical region
}

\begin{abstract}
The integration of precipitation intensity and LULC forecasting have played a significant role in prospect surface runoff, allowing for an extension of the lead time that enables a more timely implementation of the control measures. The current study proposes a full-package model to monitor the changes in surface runoff in addition to forecasting the future surface runoff based on LULC and precipitation factors. On one hand, six different LULC classes from Spot-5 satellite image were extracted by object-based Support Vector Machine (SVM) classifier. Conjointly, Land Transformation Model (LTM) was used to detect the LULC pixel changes from 2000 to 2010 as well as predict the 2020. On the other hand, ARIMA model was applied to the analysis and forecasting the rainfall trends. The parameters of ARIMA time series model were calibrated and fitted statistically to minimize the prediction uncertainty by latest Taguchi method. Rainfall and streamflow data recorded in eight nearby gauging stations were engaged to train, forecast, and calibrate the climate hydrological models. Then, distributed-GIS-based SCS-CN model was applied to simulate the maximum probable surface runoff for 2000, 2010, and 2020. The comparison results showed that first, deforestation and urbanization have occurred upon the given time and it is anticipated to increase as well. Second, the amount of rainfall has been nonstationary declined till 2015 and this trend is estimated to continue till 2020. Third, due to the damaging changes in LULC and climate, the surface runoff has also increased till 2010 and it is forecasted to gradually exceed.
\end{abstract}

Keyword: Land transformation model; ARIMA; SCS-CN; Runoff simulation; GIS 